\title{
Maternal Anxiety Affects Embryo Implantation Via Impairing Adrenergic Receptor Signaling in Decidual Cells
}

Jinxiang Wu ( $\sim$ pursuer@163.com )

the Second Affiliated Hospital of Fujian Medical University

Shu Lin

Xiamen University

Pinxiu Huang

Xiamen University

Lingling Qiu

Xiamen University

Yufei Jiang

Xiamen University

Ying Zhang

Xiamen University

Nan Meng

Xiamen University

Meiqing Meng

Xiamen University

Lemeng Wang

Xiamen University

Wenbo Deng

Xiamen University

Zhao Liu

Xiamen University

Chuanhui Guo

Xiamen University

Jinhua Lu

Xiamen University

Haibin Wang

Xiamen University

Shuangbo Kong

Xiamen University 
Article

Keywords: Recurrent pregnancy failure, psychological stress, endometrial receptivity, decidua

Posted Date: November 9th, 2021

DOI: https://doi.org/10.21203/rs.3.rs-1011033/v1

License: (c) (i) This work is licensed under a Creative Commons Attribution 4.0 International License. Read Full License

Version of Record: A version of this preprint was published at Communications Biology on August 18th, 2022. See the published version at https://doi.org/10.1038/s42003-022-03694-1. 


\section{Abstract}

Psychological stress is closely related to recurrent pregnancy failure (RPF) and infertility, but the mechanism is still unclear. The human endometrium plays a vital role in providing the site for embryo implantation; many factors have been implicated in unsatisfactory endometrial receptivity in RPF. In the current study, we found that women with pregnancy loss or infertility have a higher serum epinephrine level, indicating a potential correlation between psychological stress and pregnancy failure. RNAsequencing of the tissues from endometrial receptive phase of the normal and embryo recurrent implantation failure (RIF) and discovered that stress hormones could affect the functional status of the receptive phase of the endometrium. Subsequent analysis showed that the epinephrine signaling acts as an important regulator of endometrial receptivity through PI3K-AKT and FOXO1 signaling pathways. We also found that patients with RIF have attenuated expression of alpha-2C-adrenergic receptor (ADRA2C), and its down regulation would inhibit the promotion of adrenergic signal-mediated decidualization. We treated the early pregnancy model of stressed mice and observed that they had high serum epinephrine levels, defective uterine adrenergic receptor expression and low pregnancy rates. All together, our findings indicate that mental stress during early pregnancy can alter the functional status of endometrial receptivity.

\section{Introduction}

Psychological anxiety in women of childbearing age is one of the most common sources of infertility. Assisted reproductive technology has made some progress in the treatment of infertility. However, about $50 \%$ of pregnancies still fail clinically although the quality of the transferred embryo is good $[1,2]$. The human endometrium plays a vital role in providing the position for embryo implantation. Hence, unreceptive endometrium and abnormal early maternal-fetal interaction may lead to infertility, including repeated failure of embryo implantation. These adverse pregnancy conditions would affect the life quality of these couples as well as their psychological state.

The human endometrium is a steroid-responsive tissue that undergoes a series of regeneration cycles, including sequential proliferation, differentiation, destruction, and repair. These changes constitute the menstrual cycle [3]. This dynamic tissue remodeling ensures that the endometrium can be successfully implanted in a short time, the so-called "implantation window". During the blastocyst implantation, the endometrial stroma embeds the invasive embryos. During this process, the endometrial stromal cells undergo extensive differentiation, revascularization, and recruitment of immune cells. This process is called "decidualization" and is pertinent for a successful pregnancy [4]. Decidualization of HESCs requires correctly response to changes in peripheral hormones. The degree of decidual reaction in different species is related to the depth of the placenta, which suggests that the decidual process dominates the invasion of the trophoblast [5, 6]. In humans, decidualization is initiated regardless of pregnancy or not, and is mainly a response to ovarian steroids, such as progesterone, in the middle of the luteal phase [7, 8]. 
Recurrent implantation failure (RIF) and recurrent pregnancy loss (RPL) are common adverse reproductive events in assisted reproduction. Epidemiological data show that patients with early adverse pregnancy outcomes suffer from mental tension and anxiety $[9,10]$. Maternal stress during early pregnancy is strongly associated with various complications in the ongoing gestation [11]. Several nonrandomized control trials have reported that early pregnancy psychological support reduces the miscarriage rate in women with unexplained miscarriage $[12,13]$. However, few studies have focused on the underlying mechanisms of psychological stress associated with reproductive health, and only a handful of investigative studies have been conducted so far. In a study, the plasma epinephrine and norepinephrine levels of women with RPL were observed to be significantly higher than those of the control group [14]. Our previous study showed that maternal epinephrine exposure during early pregnancy impairs the process of uterine decidualization and embryo development in mice [15]. In the present study, we have further extended this research and have found that serum epinephrine levels are closely related to infertility and embryo implantation failure. Besides, we have confirmed that the ADRA2C is highly expressed in the decidual cells during the "implantation window" and played vital physiological functions. Herein, we revealed the mechanism of psychological stress undermining endometrial function and embryo implantation.

\section{Materials And Methods}

\section{Human subjects}

Blood samples were collected prospectively from 2018 to 2020 from patients who visited the Physical Examination Center and Reproductive Medicine Departments of The Second Affiliated Hospital of Fujian Medical University (Fujian, China). 70 blood samples were subjected to 35 women without fertility concerns from healthy physical examination (aged: 25-35 years) (control) and 35 age-matched women with infertility from reproductive assistance. Another 88 serum samples were collected from patients who undergo embryo transfer, and divided into pregnancy group and pregnancy failure group according to pregnancy outcome. The basic characteristics of the subjects are depicted in Supplemental Table 1 and 2. Serum levels of epinephrine and norepinephrine were measured via enzyme-linked immunosorbent assay (ELISA) using commercial kit (Elabscience Biotechnology Co., Wuhan, China). A total of 158 human serum samples (70 from fertility and infertility groups, and 88 from embryo transfer groups) were used for the analysis. ELISA was performed according to the manufacturer's instructions.

\section{Tissue collection}

This study involved human endometrial biopsy, as approved by The Second Affiliated Hospital of Fujian Medical University. All experiments were performed in accordance with the Declaration of Helsinki, and all volunteers provided their written informed consent before starting the study. Endometrial samples were collected from women with regular menstrual cycles (range: 28-35 days), and no significant intrauterine or ovarian abnormalities were detected by ultrasonography. After a surge in the LH was detected, the subjects underwent a biopsy during a specific period of the cycle. The pathologists at each site estimated 
the cycle day based on the standard criteria. The time of the proliferative phase sample was calculated according to the patient's cycle day, and the time of the luteal phase sample was calculated based on the subject's urine LH surge. The specimens were divided into metaphases of proliferation and secretion. Among these, 5 people who did not have uterine adhesions, inflammation, fibroids and polyps during hysteroscopy in the mid-stage proliferation were regarded as control people. In addition, 6 subjects did not have any reproductive disorders, and the cause of infertility in them was tubal obstruction or male azoospermia, another 7 subjects reported failure of repeated embryo implantation. No subject received exogenous steroid therapy in the first 3 months. These 13 subjects underwent endometrial biopsy in the mid-luteal phase. The basic characteristics of the subjects were depicted in Supplemental Table 3. The biopsies for RNA analysis were snap-frozen in liquid nitrogen and stored at $-80^{\circ} \mathrm{C}$ until use. The formalinfixed samples were embedded in paraffin and sectioned for immunohistochemistry. The histological sections were reviewed by a pathologist to verify the histological variants with reference to the criterion of Noyes [16]. Radioimmunoassay (RIA) was used to determine the serum luteinizing hormone (LH), estradiol (E2) and progesterone (P4) levels on the day of the biopsy to determine the menstrual cycle stage. These substances were evaluated by using RIA kits (Immunotech SAS, Marseille Cedex, France) according to the manufacturer's instructions.

\section{Analysis of differentially expressed mRNAs in receptivity endometrial tissues}

RNA integrity was assessed using the RNA Nano 6000 Assay Kit of the Bioanalyzer2100 system (Agilent Technologies, CA, USA). A total amount of $1 \mu \mathrm{g}$ RNA per sample was used as input material for the RNA sample preparations. Briefly, mRNA was purified from total RNA using poly-T oligo-attached magnetic beads. Fragmentation was carried out using divalent cations under elevated temperature in First Strand Synthesis Reaction Buffer (5X). First strand cDNA was synthesized using random hexamer primer and MMuLV Reverse Transcriptase (RNase H-). Second strand cDNA synthesis was subsequently performed using DNA Polymerase I and RNase H. Remain in goverhangs were converted into blunt ends via exonuclease/polymerase activities. After adenylation of 3' ends of DNA fragments, Adaptor with hairpin loop structure were ligated to prepare for hybridization. In order to select cDNA fragments of preferentially 370 420 bp in length, the library fragments were purified with AMPure XP system (Beckman Coulter,Beverly, USA). Then PCR was performed with Phusion High-Fidelity DNA polymerase, Universal PCR primers and Index $(X)$ Primer. At last, PCR products were purified (AMPureXP system) and library quality was assessed on the Agilent Bioanalyzer 2100 system. The clustering of the index-coded samples was performed on a cBot Cluster Generation System using TruSeq PE Cluster Kit v3-cBot-HS (Illumina) according to the manufacturer's instructions. After cluster generation, the library preparations were sequenced on an Illumina Novaseq platform and 150 bp paired-end reads were generated.

The differential mRNA expression between RIF and control endometrial receptivity was evaluated using the edgeR package in R/Bio conductor (version 3.26.5;

http://www.bioconductor.org/packages/release/bioc/html/edgeR.html) [17]. We used a bioconductor package complex Heatmap to generate heatmaps. The DEGs between the data sets were obtained 
using|log2-Fold Change $\geq 0.0 P$ value $\leq 0.05$ and $\mid$ log2-fold change $\mid \geq 1.0 \mathrm{P}$ value $\leq 0.05$ as cut-off criteria. To understand the DEGs underlying biological processes and pathways, Gene Ontology (GO; geneontology.org) and Kyoto Encyclopedia of Genes and Genomes (KEGG; www.genome.jp/kegg) pathway analyses were conducted using the cluster Profiler packages. The $\mathrm{GO}$ enrichment results were visualized using ggplot2 (version 3.2.0; CRAN.R-project.org/package=ggplot2). The KEGG enrichment results were analyzed by the cluster Profiler packages (version 2.1.1; CRAN.R-

project.org/package=RSQLite) and org.Hs.eg.db (version 3.8.2;

bioconductor.org/packages/org.Hs.eg.db).

\section{Mouse models}

Adult ICR mice (age: 7-8 weeks were purchased from the Vital River Laboratories Co. Ltd. All animal experiments were approved by the animal ethic committee and the Institutional Animal Care. All mice were housed in a 12-h light/12-h dark cycle (light on 07:00 AM-19:00 PM), and free water and food were provided at a temperature of $23^{\circ} \mathrm{C}$. Mice were randomly assigned to groups at the time of purchase. No data sets were excluded from the analyses. These animals were examined daily for health issues by qualified personnel; the health status was normal for all animals. The sample size, sex, and age of the animals used are specified in the text and/or figure legends.

\section{Stress model design}

The stress model test was performed in female mice pre-stressed through forced swimming in water at $15^{\circ} \mathrm{C}$ (cold water swimming) for $15 \mathrm{~min}$. Female mice were allowed to swim in a water container of $15-\mathrm{cm}$ diameter, $20-\mathrm{cm}$ height, and 11-cm depth. After swimming, the mice were gently dried by patting the body with paper towel. Repeat swimming thrice a day (from 0800 AM to 1200 AM and at 1600 PM) for 7 consecutive days. On the 7th day, the mice were allowed to mate at 18:00. Virgin females were mated with males overnight, and, in the morning (08:00 AM), the finding of the vaginal plug was designated as day 1 of pregnancy. The mice were continued to be forced to swim in cold water during the early pregnancy period. Experimental design of stressed mouse model was shown in Fig. 7A.

\section{Exogenous epinephrine addition schedules}

The mice were intravenously injected with epinephrine $(100 \mu \mathrm{g} /$ mice) (MedChem Express, Monmouth Junction, USA) in saline at 08:30 AM during days 5-7, once a day, to establish a mouse model of exogenous epinephrine in the early pregnancy period. The dosage of epinephrine was referenced or modified as used previously [15]. The mice receiving sterile saline injections served as control. Experimental design of exogenous epinephrine exposure mouse model was shown in Fig. 7G.

\section{Artificial decidualization treatment schedules}

Pseudopregnancy was produced by mating females with vasectomized male mice of the same strain (Day $1=$ vaginal plug). To induce the decidual cell response, sesame oil $(25 \mu \mathrm{L})$ was infused into a uterine horn on day 4 of pseudopregnancy; and the non-infused contralateral horn served as the control. The mice were injected with epinephrine $(100 \mu \mathrm{g} /$ mice $)$ intraperitoneally $15 \mathrm{~min}$ after the intraluminal 
infusion of oil. After that, the artificially induced decidualization mouse models were injected with epinephrine every $24 \mathrm{~h}$, and sacrificed till $72 \mathrm{~h}$. The animals in the control group only received the vehicle. Uterine weights of the infused and non-infused horns were recorded $72 \mathrm{~h}$ later. The animals in the control group only received the vehicle. Experimental design of artificially induced decidualization mouse model was shown in Fig. 7H.

\section{Tissue collection}

The uteri were obtained from the sacrificed mice of models. Before sacrificed, serums were collected from stress mouse models. Serum levels of epinephrine and norepinephrine were measured via enzyme-linked immunosorbent assay (ELISA) using mouse kit (Elabscience Biotechnology Co., Wuhan, China). After washing in physiological saline thrice, the implanting sites were frozen and stored in liquid nitrogen until RNA and protein extraction and in situ hybridization analyses, respectively. In addition, a portion of tissues was fixed in $4 \%$ (wt/vol) paraformaldehyde in $10 \mathrm{mM} \mathrm{PBS}(\mathrm{pH}=7.4)$ for histological examination.

\section{Immortalized HESCs culture and treatments}

The immortalized human endometrial stromal cell line was purchased from the American Type Culture Collection (ATCCRCRL-4003TM) and cultured according to the manufacturer's instructions. HESCs were cultured in phenol red-free DMEM/F-12 (Sigma-Aldrich, USA) containing 10\% (vol/vol) charcoal-stripped fetal bovine serum (CS-FBS) (Gibco, Grand Island, USA), $3.1 \mathrm{~g} / \mathrm{L}$ glucose [18] and $1 \mathrm{mM}$ sodium pyruvate [18], supplemented with $1.5 \mathrm{~g} / \mathrm{L}$ sodium bicarbonate [18], $50 \mathrm{mg} / \mathrm{mL}$ penicillin streptomycin (Gibco), $1 \%$ insulin-transferrin-selenium (ITS) (Gibco), and $500 \mathrm{ng} / \mathrm{mL}$ puromycin [18]. Cell cycle synchronization of HESCs was achieved via serum starvation, and the cells were treated with a medium containing $0.5 \%$ CSFBS for $12 \mathrm{~h}$. After cell starvation, the culture medium is replaced with other medium for further study.

The cell proliferation status was measured as a function of 3-(4, 5-dimethylthiazolyl-2)-2, 5diphenyltetrazolium bromide oxidation (MTS Cell Proliferation Assay Kit) in accordance with the manufacturer's instructions. The proliferation was measured with the optical density (OD) at $490 \mathrm{~nm}$ on a Synergy-HT plate reader (Bio-Tek Instruments). The experiments were performed on 3 separate occasions. In order to induce stromal cell decidualization in vitro, the cells were treated with a medium containing 2\% CS-FBS, 1 mM Medroxy progesterone 17-acetate (MPA; Sigma), and 0.25 mM dibutyryl cAMP (db-cAMP; Sigma), as previously described [19]. The media was refreshed every $48 \mathrm{~h}$, and the cultures were maintained for up to 6 days. In order to study the role of epinephrine-receptor signal in decidualization of stromal cells, we added different concentrations $(0.0 \mu \mathrm{M}, 1.0 \mu \mathrm{M}, 5.0 \mu \mathrm{M}, 10.0 \mu \mathrm{M})$ of epinephrine into the HESCs in vitro differentiation system and selected the optimal concentration for further analyses. Different concentrations of epinephrine were added at the same time as the decidualization induced.

In the RNA interference experiments, 20 mM siRNAs were transfected by Lipofectamine RNAiMAX (Invitrogen, California, USA) according to the manufacturer's instructions. Treatment with $5.0 \mu \mathrm{M}$ epinephrine in the complete medium was performed $6 \mathrm{~h}$ after the transfection, and the cells were collected after $96 \mathrm{~h}$ for RNA or protein extraction. The sequences of the siRNA were ADRA2C-siRNA target 
sequence1: 5'-GGATTTCCGGCGATCCTTT-3', ADRA2C-siRNA target sequence2: 5'-

TCAACGACGAGACCTGGTA-3', ADRA2C-siRNA target sequence3: 5'-TGTTTTGCACCTCGTCGAT-3'; and Control siRNA Med GC was used as the negative control. These three different siRNAs have the same working concentration.

\section{Real-time RT-PCR analysis}

Total RNA was extracted from cultured HESCs by using TRIZOL (Invitrogen) following the manufacturer's protocol. The same input RNA was used for all experiments. The range of RNA concentrations before reverse transcription to cDNA indifferent experiments was 1-3 $\mathrm{mg}$. The expression levels of different genes were validated by real-time RT-PCR analysis using the ABI 7500 Sequence Detector system according to the manufacturer's instructions (Applied Biosystems, Waltham Massachusetts, USA). All primers used for the real-time PCR in the present study are listed in Supplementary Table 4. The assays were performed at least thrice, each time in duplicate. Details pertinent to the bulk RNA-sequencing conducted are provided in supplementary data 1.

\section{Immunostaining}

The immunohistochemistry analysis was performed as described elsewhere [20]. The specific ADRA2C antibody (1:200; Novus, Colorado, USA) were used in 5-mm-thick paraffin embedded sections. A Histostain-SP Kit (Zhong shan Golden Bridge Biotechnology, Beijing, China) was used to visualize the antigen. For immunofluorescence, HESCs were fixed with $4 \%$ paraformaldehyde [21] in phosphatebuffered saline (PBS) at the room temperature for $10 \mathrm{~min}$. After washing with PBS, these cells were permeabilized by $0.2 \%$ Triton X-100 in PBS for 10 min and blocked with $0.5 \%$ bovine serum albumin (BSA) for $1 \mathrm{~h}$ at room temperature. Anti-ADRA2C (Abcam), Ki67 (1:200; Abcam), and FOX01 (1:200; CST, Danvers, USA) primary antibodies were respectively used.

\section{Western blotting}

Protein extraction and western blotting were performed as described previously [20]. ADRA2C (1:500), PRL (1:250; Abcam), IGFBP-1 (1:1000; Abcam), PKA (1:1000; CST, Danvers, USA), p-PKA (1:1000; CST), ERK (1:1000; CST), p-ERK (1:1000; CST), AKT (1:1000; CST), p-Thr (308) AKT (1:1000; CST), p-Ser (473) AKT (1:1000; CST), HOXOA10 (1:1000; Santa Cruz, California, USA), STAT3 (1:1000; CST), FOXO1 (1:1000; CST), GAPDH (1:2000; CST), H3 (1:2000; Abmart, Shanghai, China), and STAT3 (1:1000; CST) were used respectively. GAPDH and $\mathrm{H} 3$ served as the loading control. The bands were visualized using a chemiluminescent substrate (Super signal West Pico; Thermo Scientific) according to the manufacturer's instructions. The intensity of the bands was determined by using the Quantity One software, and the quantitative analyses of the gray-scale value of each target protein versus that of individual GAPDH were also performed.

\section{Statistical analysis}

All values are shown as the means \pm SEM or means \pm SD of at least 3 independent experiments. The parametric test was performed. Statistical analysis was performed using IBM SPSS Statistics 21 
program. Independent sample student's t-test was performed for the comparison of means. The difference was regarded as statistically significant if the two-tailed P-value was $<0.05$.

\section{Results}

\section{Serum epinephrine and norepinephrine were significantly elevated in women with infertility and pregnancy failure}

Given the strong association between mental stress and infertility, we attempted to test whether the serum levels of stress-related hormones differed in infertile women. Since epinephrine and norepinephrine are crucial stress hormones $[22,23]$, we tested the levels of these hormones in patients with and without infertility. The levels were found to be significantly higher in women with infertility than in the controls (Fig. 1A, 1B). In patients with infertility problem who undergo the embryo transfer, the serum epinephrine and norepinephrine levels of those with failed embryo implantation were higher than those with pregnancy; and the difference in epinephrine level was particularly significant (Fig. 1C, 1D). Since all the patients were transferred with good quality embryos, human endometrial receptivity dysfunction may a main cause for pregnant failure.

Analysis of the transcriptome data from 7endometrial samples with RIF and 6control samples revealed that 2511 mRNAs were differentially expressed (1260 down-regulated and 1251 up-regulated, log2-fold change $>=0.0$ and $P$ value $\leq 0.05$ as the cut-off criteria) (Fig. 1E). The detailed information for differential mRNA is presented in Data $\mathrm{S} 1$. These results demonstrated that the gene profiles of the endometrium with RIF were significantly different from those of control. Next, we applied GO analysis to predict the functions of aberrantly expressed genes. Of these DEGs, 378 mRNAs were down-regulated, while 240 mRNAs were up-regulated, with $\mid \log 2$-fold change| $\geq 1.0$ and $P$ value $\leq 0.05$ as the cut-off criteria. The biological processes of $\mathrm{GO}$ are shown in Figure 1F. The up-regulated genes were mainly involved in translation and cell proliferation, and the down-regulated genes were associated with immune regulation and inflammatory response. Immune and inflammatory signals have been confirmed to be important for embryo implantation and early pregnancy [24, 25](Fig. 1F).

\section{The adrenergic receptor ADRA2C was highly expressed in decidualized HESCs}

It is estimated that embryo quality accounts for one-third of implantation failures, while unsatisfactory endometrial receptivity and changes in embryo-endometrial dialogue are responsible for the remaining two-thirds [26, 27]. We then assessed whether adrenergic receptor signaling is involved in human endometrial receptivity. We generated heatmaps to reveal the transcriptomic patterns of adrenergic receptors (Fig. 2A), and found that only ADRA2C was highly expressed in the mid-secretory phase. However, when compared with the control group, the transcription level and protein level of ADRA2C in RIF was significantly reduced (Fig. 2A-B). We further checked the expression of ADRA2C in the human endometrium and found that it was significantly enhanced during the endometrial secretory phase (Fig. 2C-E). While investigating the cellular localization of ADRA2C in human endometrium, as shown in 
Fig. 2C, staining for ADRA2C was low or undetectable in proliferative specimens and positive in the secretory stage. We found that ADRA2C was highly expressed in glandular epithelial cells, luminal epithelial cells and stromal cells in the receptive endometrium.

Since the ADRA2C receptor was highly expressed in stroma cells and decidualization of HESCs is crucial for the establishment of endometrial receptivity, we utilized an immortalized human endometrial stromal cell line, which can be induced to decidualization to employ the function of adrenergic signal for endometrium [15]. We screened the expression profiles of different adrenergic receptors using RT-PCR during decidualization, and only ADRA2C exhibited an enhanced expression pattern during the in vitro decidualization (Fig. 2F-I). Moreover, we detected phenylethanolamine N-methyltransferase (PNMT) and tyrosine hydroxylase $(\mathrm{TH})$, which are key rate-limiting enzymes for the synthesis of adrenergic ligands[28, 29]. The results signified that these adrenergic receptors were present in the human endometrium, however, HESCs could not synthesize epinephrine ligands per se(Supplemental Fig. S1A-B).

\section{Adrenergic Signaling Is Involved In The Decidualization Of Hescs}

To elucidate the role of ADRA2C in the decidualization of HESCs, we examined the expression level of classical decidualization markers, including prolactin $(P R L)$ and insulin-like growth factor-binding protein 1 (IGFBP-1), in different concentrations of epinephrine-treated HESCs. We found that only $5.0 \mu \mathrm{M}$ epinephrine effectively promoted decidualization (Fig. 3A-3B) and that this promotion was time dependent (Fig. 3C-3D).In addition, $10 \mu \mathrm{M}$ adrenaline loss the promoting effects compared with $5 \mu \mathrm{M}$ (Fig. 3A-B, Fig. 3E-F). Since ADRA2C was weakly expressed in undifferentiated HESCs, we used 0-10.0 $\mu \mathrm{M}$ epinephrine for cell growth analysis to evaluate its effect on HESCs culture in vitro. As shown in Supplemental Fig. S2, different concentrations of epinephrine display little effects on the proliferation activity of HESCs. Therefore, we used the exposure dose of $5 \mu \mathrm{M}$ epinephrine for further studies.

\section{Adrenergic signaling inhibits AKT activity to increase Forkhead box 01 (FOX01) expression and nuclear localization}

The observation that appropriate level of adrenergic signal promotes the HESC decidualization motivate us to explore the underlying mechanism for $A D R A 2 C$ regulating the normal process of human endometrial stromal differentiation. ADRA2C belongs to G-protein-coupled receptors (GPCRs), and its common downstream activation pathways include PKA, ERK, and AKT [30]. Western blotting was conducted to detect the expression level of these signal molecules. Only the AKT signaling pathway was affected by epinephrine stimulation, and the phosphorylation level of p-AKT (Ser473) was reduced by 5 $\mu \mathrm{M}$ epinephrine stimulation, but not in $10 \mu \mathrm{M}$ group (Fig. 4A). It was also noticed that the ADRA2C receptor as down-regulated in the high $(10 \mu \mathrm{M})$ epinephrine treated group (Fig. 4A). A wealth of data indicated that AKT pathway activity was involved in decreased FOXO1 protein and blunted the decidual response [31,32]. Furthermore, as expected, the level of FOXO1 protein increased with the decrease in AKT phosphorylation level (Fig. 4A and 4B). We also assayed other transcription factors such as HOXA10 and STAT3, which were key mediators in decidualization [33-35]. Interestingly, the protein level of factor HOXA10 is increased by epinephrine stimulation but seem not to be affected by epinephrine 
concentration and the protein level of ADRA2C (Fig. 4B). We found that the change of FOXO1 was affected by epinephrine concentration; moreover, these changes were affected by AKT phosphorylation

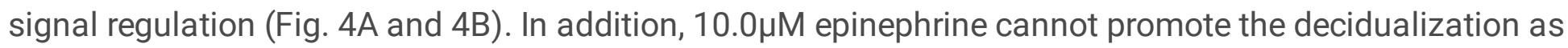
the $5.0 \mu \mathrm{M}$, consistent with the inability to increase the FOXO1 level. Based on these observations, it seems that the physiological level of epinephrine is benefit for decidualization through its receptor ADRA2C, and aberrantly elevated epinephrine loss of its promoting effects for decidualization. During decidualization, FOXO1 is responsible for transcribing the IGFBP-1 and PRL genes [36, 37]. FOXO1 might be compartmentalized in both the cytoplasm and nucleus. Nonetheless, it must be located in the nucleus to perform transcriptional activity. Hence, we next used western blotting and immune fluorescence experiments to detect the distribution of FOXO1 in the cytoplasm and nucleus of HESCs. Indeed, the

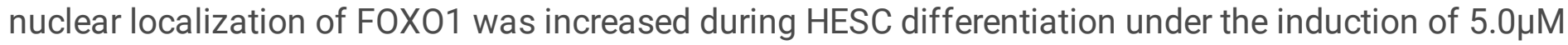
epinephrine, but not $10.0 \mu \mathrm{M}$ (Fig. 4C-E). Collectively, these results suggested that ADRA2C activation was an important process in assisting the decidualization of HESCs, and high concentrations of epinephrine restrain the process of HESCs decidualization.

\section{Aberrant Adra2c Signaling Hampered Hescs Decidualization}

To verify whether the clearance of the accumulated ADRA2C would reverse the effects of assisting in HESCs decidualization, we employed a small interference RNA (siRNA) technique to knockdown the expression of $A D R A 2 C$ in HESCs. As shown in Fig. 5A-B and Supplemental Fig. S3A, during the decidualization process, ADRA2C-siRNA largely reduced ADRA2C expression at both the mRNA and protein levels in the HESCs. This decreased expression can partially eliminate the synergistic effect of adrenergic signals on the decidualization of HESCs. Real-time quantitative PCR was used to evaluate the decidualization markers IGFBP-1 and PRL (Fig. 5C-D). Consistent with this observation, knockdown of $A D R A 2 C$ expression also largely enhanced the activity of AKT signaling. This increase lowered the level of FOXO1 protein, which attenuated the effects of adrenergic signaling to promote the decidualization of HESCs (Fig. 5E). Then, we tested whether ADRA2C knockdown would reduce the nuclear localization of FOX01. As shown in Fig. 5F and Supplemental Fig. S3B, when compared with the control group, the level of FOXO1 protein and the localization of FOXO1 in the nucleus of HESCs were both reduced after ADRA2C knockdown. These findings further strengthen the view that ADRA2C signaling and HESCs decidualization were functionally associated. Overall, these experiments suggest that epinephrine could promote HESC decidualization by inhibiting the AKT activity to increase FOXO1 expression and nuclear localization via ADRA2C (Fig. 5G).

\section{Altered expression of ADRA2C and FOXO1 in the endometrium during the implantation window from RIF patients}

Our previous study found that when compared with the endometrial proliferation phase, the level of ADRA2C protein in the endometrial secretory phase was increased. Moreover, we found that an appropriate dose of epinephrine could promote the differentiation of HESCs. Interestingly, we discovered that high concentrations of epinephrine did not promote the differentiation of HESCs in vitro, but down- 
regulate the expression of its receptor ADRA2C (Fig. 4B). Since the epinephrine level of the RIF group was higher than that of the control group, we checked whether endometrium from the mid-luteal phase of the menstrual cycle displayed the similar alternation as observed in vitro. We identified that $A D R A 2 C$ was involved in significantly differentially down-regulated genes, as displayed in the volcano map (Fig. 6A). Next, we used pathway analyses to identify the significant pathways associated with the DEGs according to Kyoto Encyclopedia of Genes and Genomes (KEGG) (Fig. 6B). KEGG pathway analysis revealed that Akt signaling was involved in the affected pathway among the up-regulated genes (Fig. 6B). In addition, WB data showed that the protein level of ADRA2C in the mid-luteal phase of the menstrual cycle from RIF patients was lower than that of controls (Fig. 6C), and expression and distribution patterns of ADRA2C and FOXO1 in the endometrium of RIF patients were more heterogeneous compared with those in controls (Fig. 6D-E). We also noted that the expression patterns of ADRA2C, FOX01, and PR, which were considered to be the genes related to endometrial receptivity [37, 38], were more consistent in terms of the expression level and spatial location in the normal individuals (Fig. S4A-B). Therefore, consistent with these findings, we hypothesized that serum epinephrine levels may alter the endometrial receptivity by affecting the expression of adrenal receptors and thus the downstream signal cascade involved AKTFOXO1 in the endometrium.

\section{High epinephrine down regulated the uterine adrenergic receptor and compromised the function of decidual cells in mice}

To further verify the detrimental effects of high level of adrenergic ligand on decidualization and early pregnancy in vivo, a mouse stress model was utilized (Fig. 7A). Stress-induced mice had increased serum epinephrine levels (Fig. 7B-C), significantly decreased mating rate (Fig. 7D), and poor embryonic development (Fig. 7E-F). As shown in Fig. 7F, the arrow points out growth-restricted implantation sites on D6 and D8 of stressed mouse models. Our previous studies had confirmed that mouse decidual cells in early pregnancy express $A d r a 1 b, A d r a 2 b$, and $A d r b 2$ receptors [15]. We speculated that these impairments of decidualization may be related to abnormal adrenergic receptor signaling. Subsequently, through in situ and quantitative PCR detection, we found that the expressions of these adrenergic receptors in the decidual cells of the stressed mice were significantly reduced (Fig. 7K-0). Moreover, exogenous epinephrine decreased adrenergic receptor expression in uterine decidual cells during early pregnancy (Fig. 7K-N). We use the artificially induced decidualization mouse model (Fig. 7H) and found that exogenous epinephrine exposure can impair the decidual differentiation of endometrial stromal cells (Fig. 7l-J). This indicated that the significantly decreased expression of these receptors was not caused by abnormal embryonic development. These interesting findings suggest that elevated epinephrine in vivo impaired adrenergic receptor signaling, disrupted the decidualization of uterine stromal cells leading to early pregnancy loss.

\section{Discussion}

In this study, our main findings include: increased serum epinephrine levels are closely related to infertility and embryo implantation failure; during the "implantation window," epinephrine receptor ADRA2C is 
highly expressed in the stromal or decidual cells; appropriate activation of ADRA2C signal can reduce the phosphorylation level of AKT, upregulate the level of FOXO1 protein and its nuclear localization, thereby promoting the decidualization of endometrial stromal cells; RNA-Seq performed with endometrium from the receptive phase revealed the dysfunctional state of the endometrium associated with stress status in RIF patients; Serum epinephrine level was higher and the pregnancy rate was lower in stressed mice, which displayed the defective expression of uterine adrenergic receptor and abnormal decidua. In short, by multiple approaches, the molecular mechanism was uncovered that psychological stress directly disrupts endometrial functioning and early embryo implantation.

A recent study also reported that exogenous epinephrine and norepinephrine could affect the development of mouse uterine decidua and early embryonic development $[15,39]$. The previous studies have confirmed that excessive exogenous epinephrine can cause signal abnormalities during decidual development, including Ptgs2 (encoding COX2), Wnt4 and Bmp2 signaling. In addition, several critical transcriptional factors during decidualization, including FOX01, Meis Homeobox 1 (MEIS1) and CCAAT Enhancer Binding Protein Beta (CEBPB) were decreased. Exogenous epinephrine exposure during early pregnancy resulted in impaired decidualization and early pregnancy in mice [15]. RNA-Seq data showed that ADRA2C was highly expressed in the human endometrium, signifying the underlying physiological function of adrenergic receptor signaling in human endometrium. Furthermore, we observed that ADRA2C signaling was present in endometrial stromal cells. Decidualization refers to the transformation of endometrial stromal fibroblasts into characteristic secretory decidual cells that provide nourishment and an immune-privileged matrix for embryo implantation and placental development [40]. In spite of the clinical importance of decidualization, the mechanisms are yet to be elucidated in vivo owing to its complexity. Few studies have investigated whether neuropsychiatric factors are involved in the establishment of a functional endometrium, especially during endometrial stromal cell decidual differentiation. In the present work, we have confirmed the presence of adrenergic neurotransmitters and their receptors in the endometrium and have shown that they have a physiological regulatory significance in establishing endometrial receptivity.

From a mechanistic point of view, we found that the decidualization of endometrial stromal cells and the gradual increase of ADRA2C expression, resulting in a decrease in phosphorylated AKT and thus FOXO1 nuclear translocation. The more nuclear localized FOXO1 leads to an increase in IGFBP-1 and PRL mRNA levels under epinephrine stimulation (Fig. 4). As mentioned earlier, FOXO1 is a transcription factor that also plays an important role in the process of decidualization, which is necessary for endometrial receptivity and can regulate theexpression of genes encoding IGFPB-1 and PRL $[37,40]$. In mammalian cells, AKT phosphorylates Ser256 to inhibit FOX01 activity, leading to its nuclear export and transcriptional inactivation [41]. Moreover, it has been demonstrated that FOXO1 is dephosphorylated during phosphatidic acid-induced decidualization of HESCs by inactivating AKT via the AKT-PP2A complex [31]. This theory is supported by our observation that the knockdown of ADRA2C increases AKT phosphorylation and decreases FOX01 protein levels in the decidual cells (Fig. 5). Even though the findings of this study are somewhat consistent with previous results, further experiments are needed to ascertain the complexes recruited by adrenergic signals that lead to AKT dephosphorylation (Fig. 4-5). 
To analyze the molecular cascade of events leading to increased disease susceptibility, we used a stressed mouse model [42]. Stress stimulation can lead to increased serum adrenaline levels, thereby impairing sexual mating and early embryonic development in mice (Fig. 7B-F). Preliminary work has confirmed that $A d r a 1 b, A d r a 2 b$ and $A d r b 2$ adrenergic receptors were specifically and highly expressed in the mouse decidual cells at early pregnancy. Interestingly, we found that in mouse models with increased endogenous and exogenous epinephrine, the expressions of $A d r a 1 b, A d r a 2 b$ and $A d r b 2$ adrenergic receptors in the decidual cells were decreased significantly, which was also confirmed in the oil induced artificial decidual model. Our previous studies have confirmed that maternal epinephrine exposure during early pregnancy inhibited the proliferation of endometrial stromal cells and key modulators of decidualization, including COX2, BMP2, and WNT4 [15]. Hence, it was likely that the decidualization function impaired by exogenous epinephrine was closely related to the aberrant adrenergic receptor signaling in mouse uterus. Collectively, the in vivo and in vitro experiments have demonstrated that adrenergic receptor signaling pathway was involved in the physiological and pathological process of endometrial stromal cells differentiation.

Glucocorticoid (GC) is a stress-induced steroid hormone that is released from the adrenal cortex and is essential for stress adaptation. Glucocorticoid receptor(GR) in the mouse uterus is essential fornormal fertility. The deficiency in uterine GR signaling resulted in an exaggerated inflammatory response to induced decidualization, including altered immune cell recruitment [43]. However, stress-induced GCs exposure has an adverse effect on decidualization and uterine receptivity by causing collagen disorders [44]. These results demonstrate that stress-related hormones and associated receptors have both physiological functions and pathological damage during early pregnancy. Our data also indicated that the appropriate epinephrine is beneficial to HESCs decidualization, but aberrantly elevated epinephrine has a detrimental effect on early pregnancy.

Taken together, our study has offered new insights into the mechanism of adrenergic signaling in human endometrial functioning and has provided a firm experimental basis for future research on the relationship between psychological stress factors and early adverse pregnancy outcomes.

\section{Declarations}

\section{Conflicts of Interest}

There is no conflict of interest to declare.

\section{Funding}

This work was supported by National Natural Science Foundation of China (81901481 to J.W.).

\section{Author contributions}

Jinxiang Wu, Shuangbo Kong and Haibin Wang designed the experiments. Jinxiang Wu and Pinxiu Huang performed the experiments. Jinxiang Wu and Yufei Jiang performed statistical analysis and wrote 
the manuscript. Shu Lin and Lingling Qiu collected the human endometrial tissues. All authors reviewed the manuscript.

\section{Ethic/Informed Consent Statement}

The research was approved by the Biomedical Research Ethics Review Committee of Fujian Medical University ([2019] Fuyi Ethics Review No. (101)) and does not violate ethical principles and agrees to implement the research under the premise of informed consent. According to the requirements of the local ethics committee, the research meets the ethical approval point of view. All participants received full informed consent.

\section{References}

1. El-Danasouri, I., et al., Effect of transferring a morphologically impaired embryo with a good quality embryo on the pregnancy and implantation rates. Eur Rev Med Pharmacol Sci, 2016. 20(3): p. 3948.

2. Otani, T., et al., Preimplantation genetic diagnosis significantly improves the pregnancy outcome of translocation carriers with a history of recurrent miscarriage and unsuccessful pregnancies. Reprod Biomed Online, 2006. 13(6): p. 869-74.

3. Norwitz, E.R., D.J. Schust, and S.J. Fisher, Implantation and the survival of early pregnancy. N Engl J Med, 2001. 345(19): p. 1400-8.

4. Zhang, S., et al., Physiological and molecular determinants of embryo implantation. Mol Aspects Med, 2013. 34(5): p. 939-80.

5. Brosens, J.J., R. Pijnenborg, and I.A. Brosens, The myometrial junctional zone spiral arteries in normal and abnormal pregnancies: a review of the literature. Am J Obstet Gynecol, 2002. 187(5): p. 1416-23.

6. Ramsey, E.M., M.L. Houston, and J.W. Harris, Interactions of the trophoblast and maternal tissues in three closely related primate species. Am J Obstet Gynecol, 1976. 124(6): p. 647-52.

7. Weimar, C.H., et al., The motile and invasive capacity of human endometrial stromal cells: implications for normal and impaired reproductive function. Hum Reprod Update, 2013. 19(5): p. 542-57.

8. Dey, S.K., et al., Molecular cues to implantation. Endocr Rev, 2004. 25(3): p. 341-73.

9. Kolte, A.M., et al., Depression and emotional stress is highly prevalent among women with recurrent pregnancy loss. Hum Reprod, 2015. 30(4): p. 777-82.

10. Kagami, M., et al., Psychological adjustment and psychosocial stress among Japanese couples with a history of recurrent pregnancy loss. Hum Reprod, 2012. 27(3): p. 787-94.

11. Neugebauer, R., et al., Association of stressful life events with chromosomally normal spontaneous abortion. Am J Epidemiol, 1996. 143(6): p. 588-96. 
12. van den Berg, M.M.J., et al., Patient-centered early pregnancy care: a systematic review of quantitative and qualitative studies on the perspectives of women and their partners. Hum Reprod Update, 2018. 24(1): p. 106-118.

13. Clifford, K., R. Rai, and L. Regan, Future pregnancy outcome in unexplained recurrent first trimester miscarriage. Hum Reprod, 1997. 12(2): p. 387-9.

14. Nakatsuka, M., et al., Increased plasma adrenomedullin in women with recurrent pregnancy loss. Obstet Gynecol, 2003. 102(2): p. 319-24.

15. Wu, J., et al., An exaggerated epinephrine-adrenergic receptor signaling impairs uterine decidualization in mice. Reprod Toxicol, 2019. 90: p. 109-117.

16. Noyes, R.W. and J.O. Haman, Accuracy of endometrial dating; correlation of endometrial dating with basal body temperature and menses. Fertil Steril, 1953. 4(6): p. 504-17.

17. Robinson, M.D., D.J. McCarthy, and G.K. Smyth, edgeR: a Bioconductor package for differential expression analysis of digital gene expression data. Bioinformatics, 2010. 26(1): p. 139-40.

18. Zhai, X., et al., Cisplatin-DNA adducts inhibit ribosomal RNA synthesis by hijacking the transcription factor human upstream binding factor. Biochemistry, 1998. 37(46): p. 16307-15.

19. Krikun, G., et al., A novel immortalized human endometrial stromal cell line with normal progestational response. Endocrinology, 2004. 145(5): p. 2291-6.

20. Lu, J., et al., A positive feedback loop involving Gcm1 and Fzd5 directs chorionic branching morphogenesis in the placenta. PLoS Biol, 2013. 11(4): p. e1001536.

21. Pfannkuche, K., et al., Cardiac myocytes derived from murine reprogrammed fibroblasts: intact hormonal regulation, cardiac ion channel expression and development of contractility. Cell Physiol Biochem, 2009. 24(1-2): p. 73-86.

22. Boyanova, L., Stress hormone epinephrine (adrenaline) and norepinephrine (noradrenaline) effects on the anaerobic bacteria. Anaerobe, 2017. 44: p. 13-19.

23. Mahapatra, M.S., S.K. Mahata, and B. Maiti, Effect of stress on serotonin, norepinephrine, epinephrine and corticosterone contents in the soft-shelled turtle. Clin Exp Pharmacol Physiol, 1991. 18(10): p. 719-24.

24. Dekel, N., et al., The role of inflammation for a successful implantation. Am J Reprod Immunol, 2014. 72(2): p. 141-7.

25. Dekel, N., et al., Inflammation and implantation. Am J Reprod Immunol, 2010. 63(1): p. $17-21$.

26. Simón, C., et al., Cytokines and embryo implantation. J Reprod Immunol, 1998. 39(1-2): p. $117-31$.

27. Franasiak, J.M., et al., The nature of aneuploidy with increasing age of the female partner: a review of 15,169 consecutive trophectoderm biopsies evaluated with comprehensive chromosomal screening. Fertil Steril, 2014. 101(3): p. 656-663.e1.

28. Laborie, C., et al., Metyrapone-induced glucocorticoid depletion modulates tyrosine hydroxylase and phenylethanolamine $\mathrm{N}$-methyltransferase gene expression in the rat adrenal gland by a noncholinergic transsynaptic activation. J Neuroendocrinol, 2003. 15(1): p. 15-23. 
29. Kawahata, I. and T. Yamakuni, Imidacloprid, a neonicotinoid insecticide, facilitates tyrosine hydroxylase transcription and phenylethanolamine $N$-methyltransferase $m R N A$ expression to enhance catecholamine synthesis and its nicotine-evoked elevation in PC12D cells. Toxicology, 2018. 394: p. 84-92.

30. Wang, B., Y. Yang, and P.A. Friedman, $\mathrm{Na} / \mathrm{H}$ exchange regulatory factor $\mathrm{1}$, a novel AKT-associating protein, regulates extracellular signal-regulated kinase signaling through a B-Raf-mediated pathway. Mol Biol Cell, 2008. 19(4): p. 1637-45.

31. Lee, S.Y., et al., Phosphatidic acid induces decidualization by stimulating Akt-PP2A binding in human endometrial stromal cells. Febs j, 2016. 283(22): p. 4163-4175.

32. Yin, X., et al., Increased activation of the PI3K/AKT pathway compromises decidualization of stromal cells from endometriosis. J Clin Endocrinol Metab, 2012. 97(1): p. E35-43.

33. Cottrell, H.N., et al., Effects of Supraphysiologic Levels of Estradiol on Endometrial Decidualization, sFIt1, and HOXA10 Expression. Reprod Sci, 2019. 26(12): p. 1626-1632.

34. Xu, Y., et al., HOXA10 co-factor MEIS1 is required for the decidualization in human endometrial stromal cell. J Mol Endocrinol, 2020. 64(4): p. 249-258.

35. Jiang, Y., et al., FoxM1 Directs STAT3 Expression Essential for Human Endometrial Stromal Decidualization. Sci Rep, 2015. 5: p. 13735.

36. Takano, M., et al., Transcriptional cross talk between the forkhead transcription factor forkhead box 01A and the progesterone receptor coordinates cell cycle regulation and differentiation in human endometrial stromal cells. Mol Endocrinol, 2007. 21(10): p. 2334-49.

37. Kajihara, T., J.J. Brosens, and O. Ishihara, The role of FOXO1 in the decidual transformation of the endometrium and early pregnancy. Med Mol Morphol, 2013. 46(2): p. 61-8.

38. Lessey, B.A., et al., Endometrial progesterone receptors and markers of uterine receptivity in the window of implantation. Fertil Steril, 1996. 65(3): p. 477-83.

39. Wang, J., et al., Norepinephrine exposure restrains endometrial decidualization during early pregnancy. J Endocrinol, 2021. 248(3): p. 277-288.

40. Gellersen, B. and J.J. Brosens, Cyclic decidualization of the human endometrium in reproductive health and failure. Endocr Rev, 2014. 35(6): p. 851-905.

41. Lam, E.W., R.E. Francis, and M. Petkovic, FOXO transcription factors: key regulators of cell fate. Biochem Soc Trans, 2006. 34(Pt 5): p. 722-6.

42. Wang, Q., M. Wang, and M.D. Whim, Neuropeptide y gates a stress-induced, long-lasting plasticity in the sympathetic nervous system. J Neurosci, 2013. 33(31): p. 12705-17.

43. Whirledge, S.D., et al., Uterine glucocorticoid receptors are critical for fertility in mice through control of embryo implantation and decidualization. Proc Natl Acad Sci U S A, 2015. 112(49): p. 15166-71.

44. Zheng, H.T., et al., The detrimental effects of stress-induced glucocorticoid exposure on mouse uterine receptivity and decidualization. Faseb j, 2020. 34(11): p. 14200-14216. 


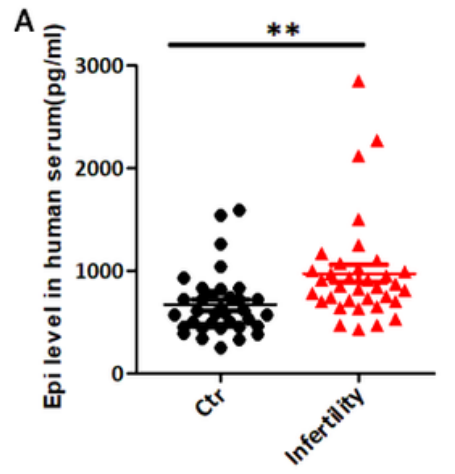

E

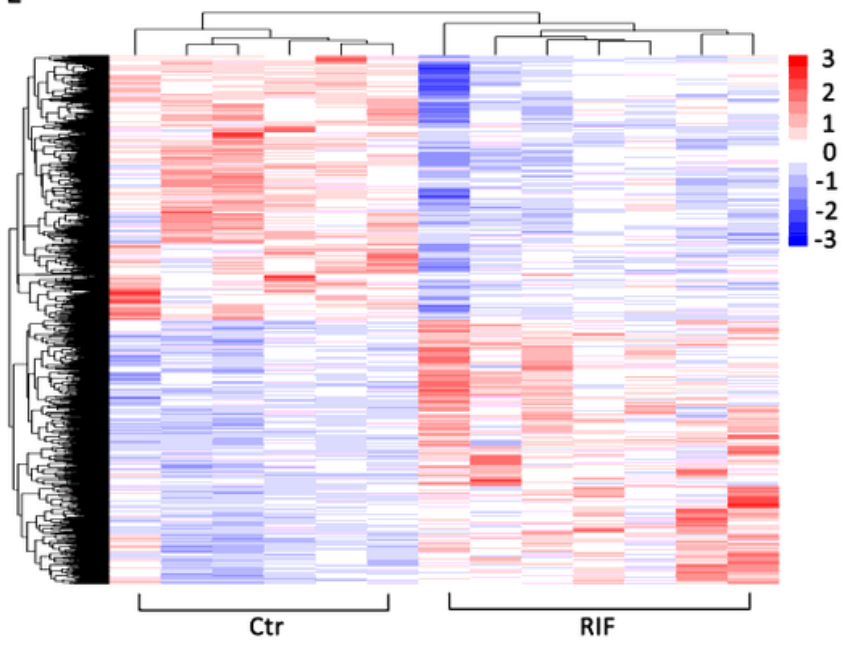

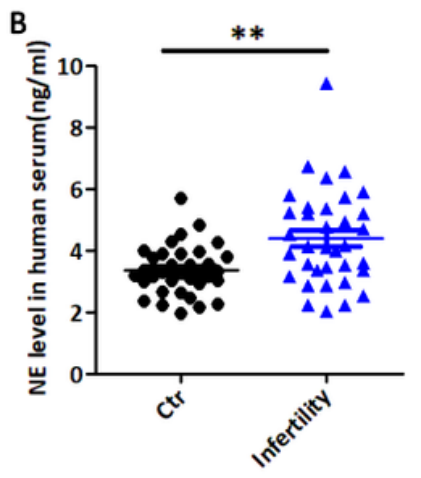

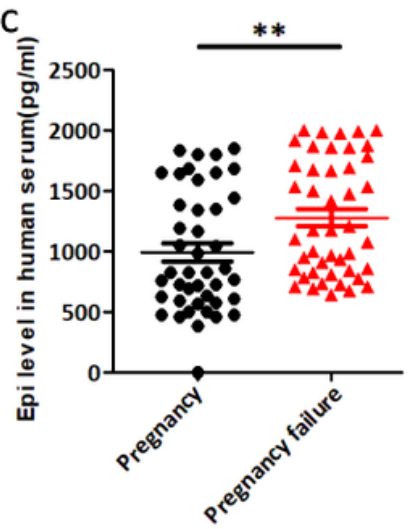

F

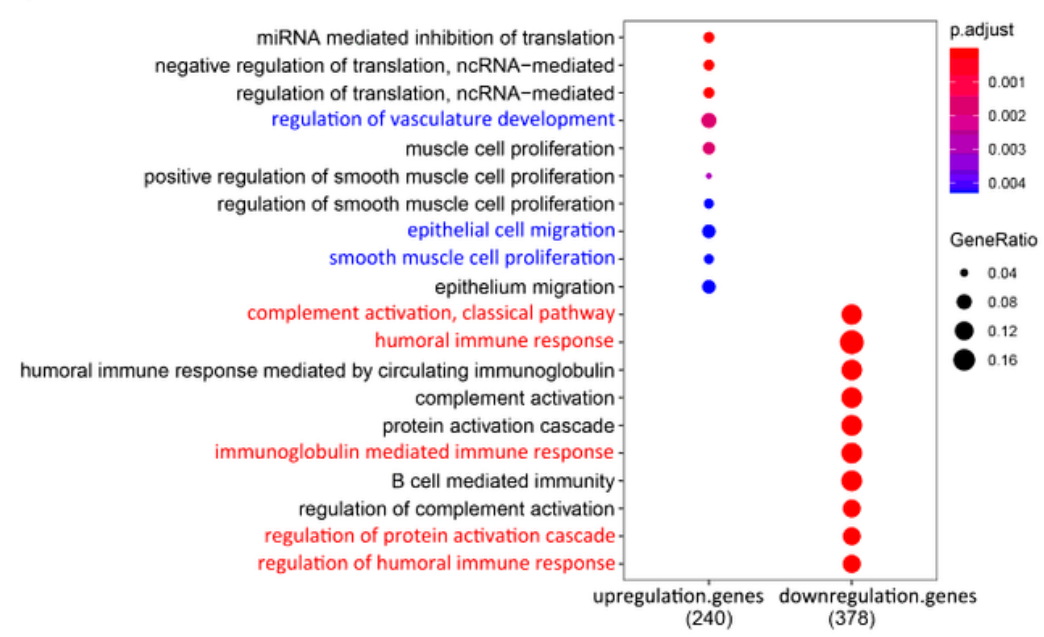

Figure 1

The changes in serum epinephrine and norepinephrine levels and transcriptomic wide differences for endometrium in RIF patients through RNA-seq analysis. $(A, B)$ Epinephrine and norepinephrine levels in control patients $(n=35)$ and infertility patients serum $(n=35) ;(C, D)$ Epinephrine and norepinephrine levels in pregnancy patients $(n=43)$ and embryo implantation failure patients serum $(n=45) ;(E)$ Heatmap of upregulated and down-regulated endometrial genes in the putitive recetivive windows in RIF compared with Ctr(upregulated genes in red and downregulated genes in blue); (F)Signifcantly enriched GO terms for the DEGs in receptivity endometrium with RIF (up-regulated genes in red and down-regulated genes in blue). Significance is indicated as - $\log 10 \mathrm{p}$ value.Ctr, control; RIF, recurrent implantation failure;Values are represented as the mean \pm SEM. For statistical analysis, $p$ values were calculated by one-way ANOVA followed by Tukey's multiple comparison post hoc test. Statistical significance for all analyses were * $\mathrm{p}<$ $0.05 ; * \star \mathrm{p}<0.01 ; * * * \mathrm{p}<0.001$ 
A

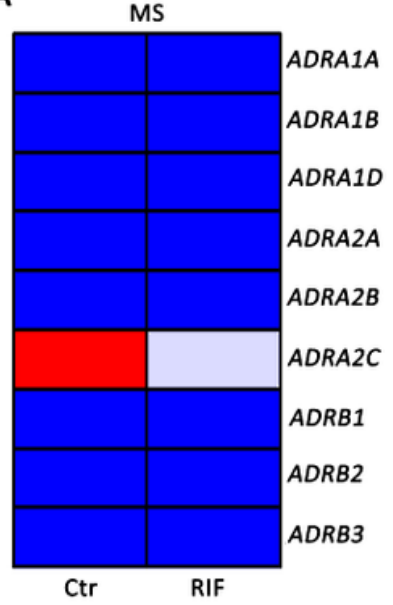

B

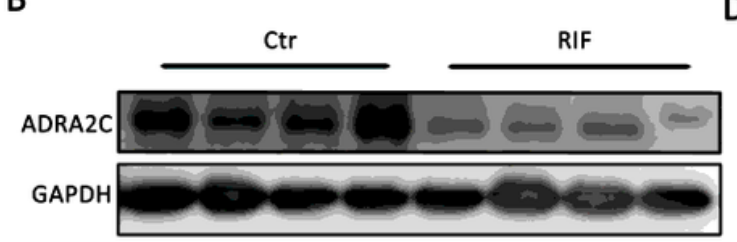

F

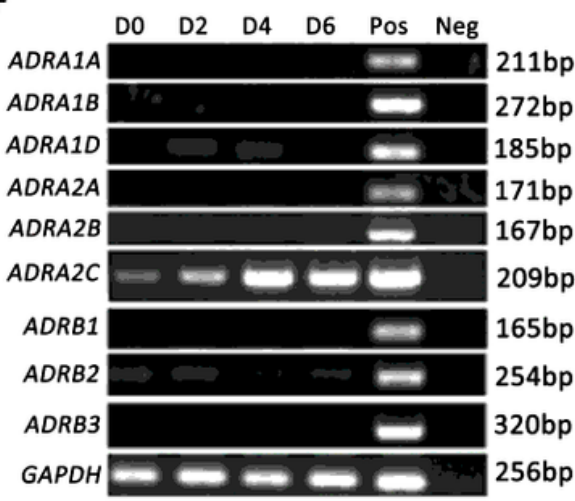

C

G
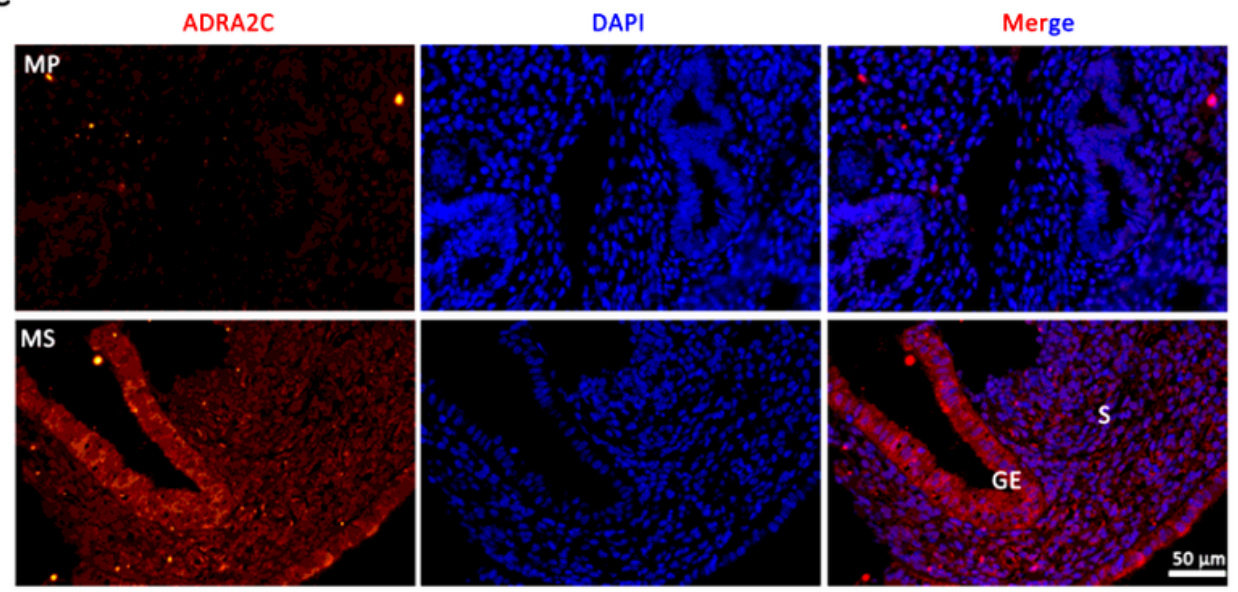

E

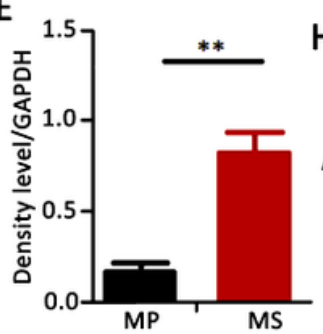

$\mathbf{H}$
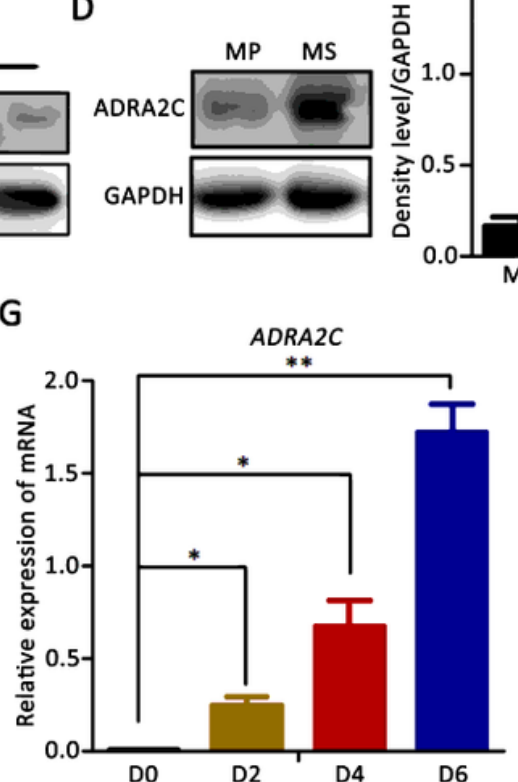

I

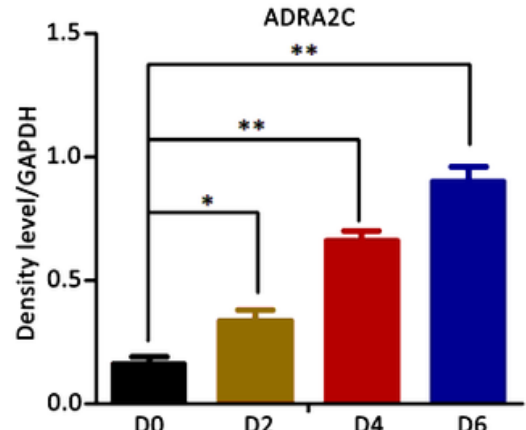

\section{Figure 2}

ADRA2C wasrobust expressed in HESCs during decidualization. (A) The heatmap showed the alterationstranscriptomic patterns of adrenergic receptors in RIF versus controlwomen (up-regulated genes in red and down-regulated genes in blue); MS, mid-secretory phase.(B) Western blotting for the ADRA2C protein levels in control and RIF women. (C) Tissue immunofluorescence for ADRA2C protein location in the Mid-proliferative stage and Mid-secretory phase of human endometrium. Scale bars, 50 um. MP, Mid-proliferative phase; MS, mid-secretory phase; S, stroma; GE, glandular epithelium; (D, E) The relative protein levels of ADRA2C between Mid-proliferative and mid-secretory phase of endometrial tissues was determined by western blot. (F) RT-PCR screen for the expression of different adrenergic receptors during decidualization of HESCs. Pos, positive control; Neg, negative control. (G) Quantitative RT-PCR analysis of ADRA2C mRNA level in HESCs during decidualization. $(\mathrm{H}, \mathrm{I})$ The relative protein levels of ADRA2C before and after HESCs differentiation was determined by western blot. D0, HESCs cultured in vitro before differentiation; D4, HESCs induced differentiation on day 4 in vitro; 
A

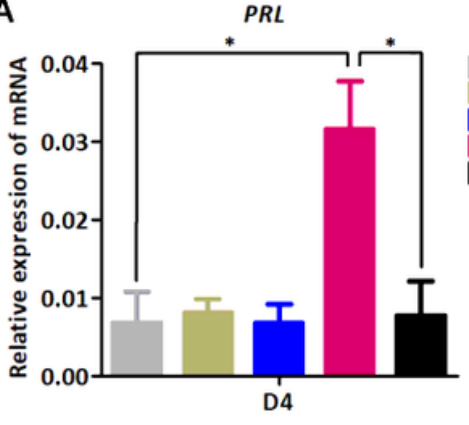

B

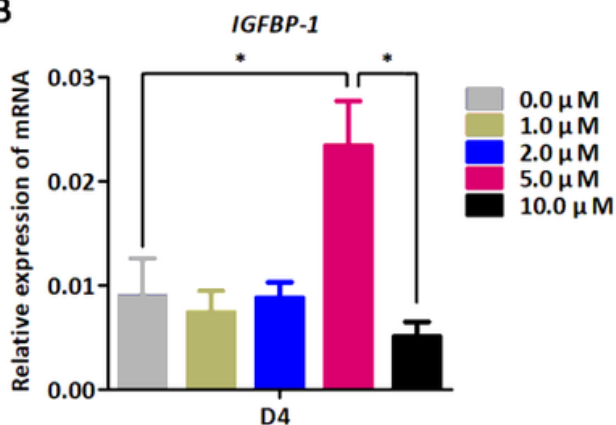

C

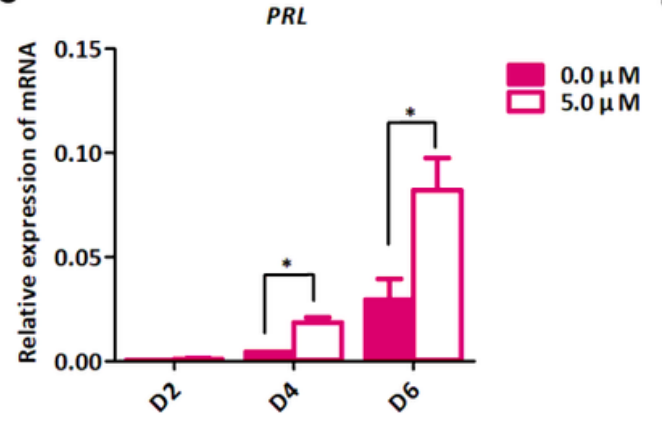

D

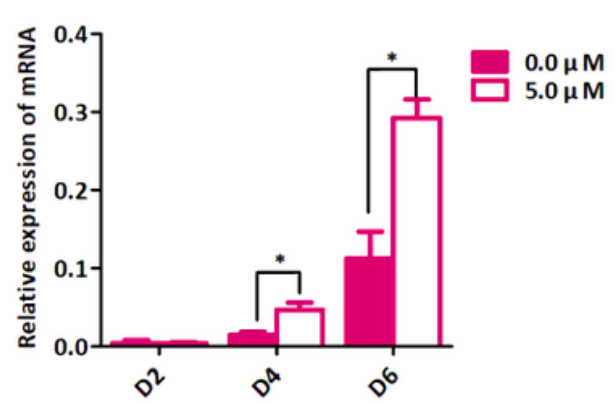

E

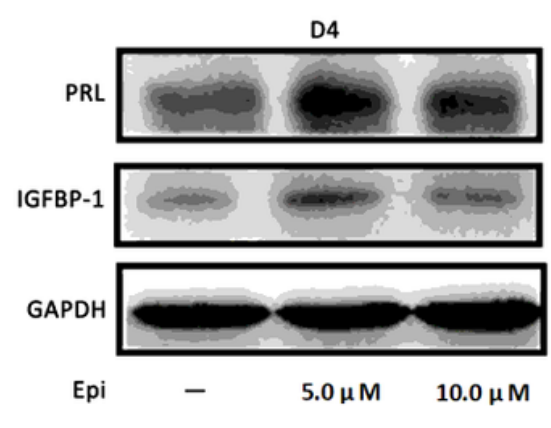

F

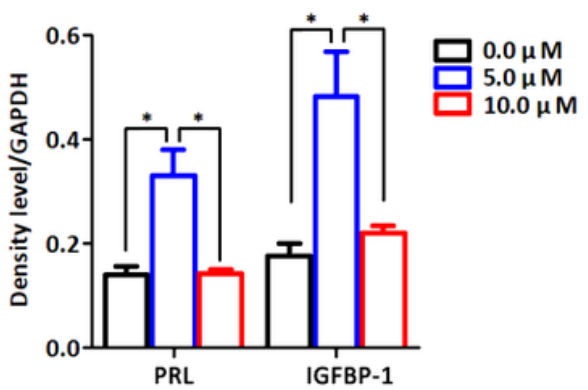

\section{Figure 3}

Dose dependent role of adrenergic signalingin HESCs during decidualization. $(A, B)$ The mRNA levels of decidualization markers prolactin (PRL) and insulin-like growth factor binding protein 1 (IGFBP-1) on the 4th day of decidualization in the control and epinephrine treated group. The values are normalized to the GAPDH expression level and indicated as the mean \pm SEM, $n=3$. Epi, epinephrine; (C, D) The mRNA levels

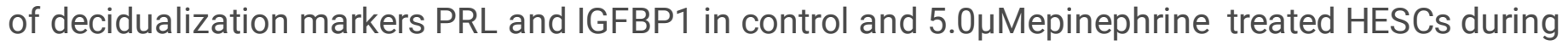
induced decidualization at different time. The values are normalized to the GAPDH expression level and indicated as the mean \pm SEM, $n=3$. Epi, epinephrine; $(E, F)$ The relative proteins levels of PRL and IGFBP-1 before and after HESCs differentiation were determined by western blot.
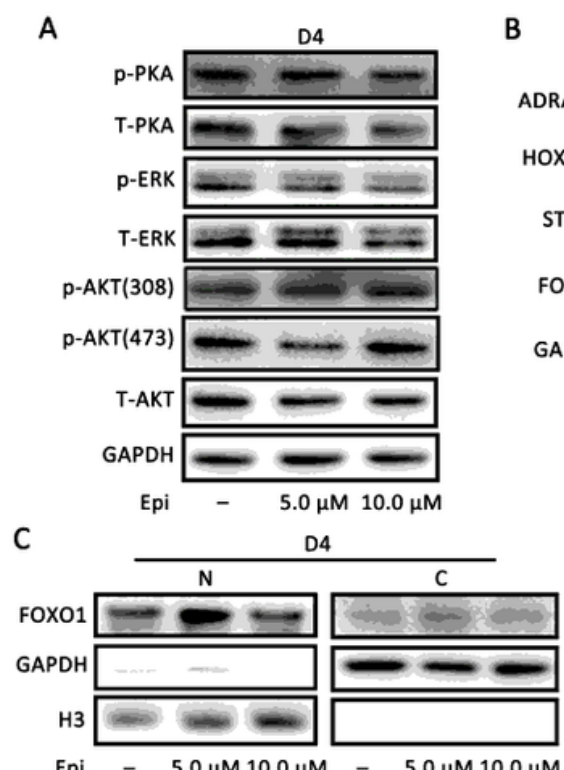

Epi $\quad-5.0 \mu \mathrm{M} 10.0 \mu \mathrm{M}-5.0 \mu \mathrm{M} 10.0 \mu \mathrm{M}$
B

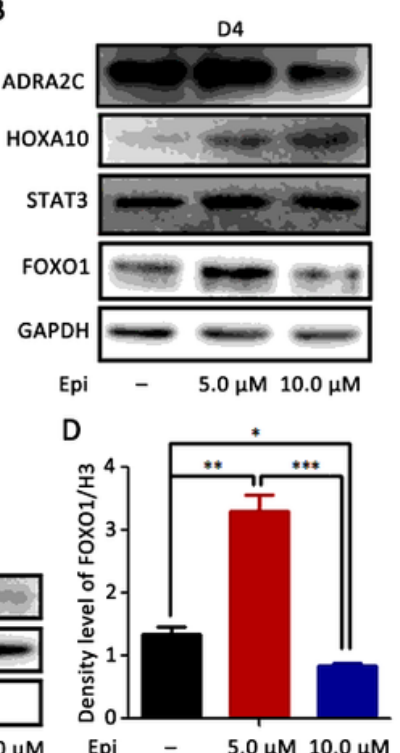

E

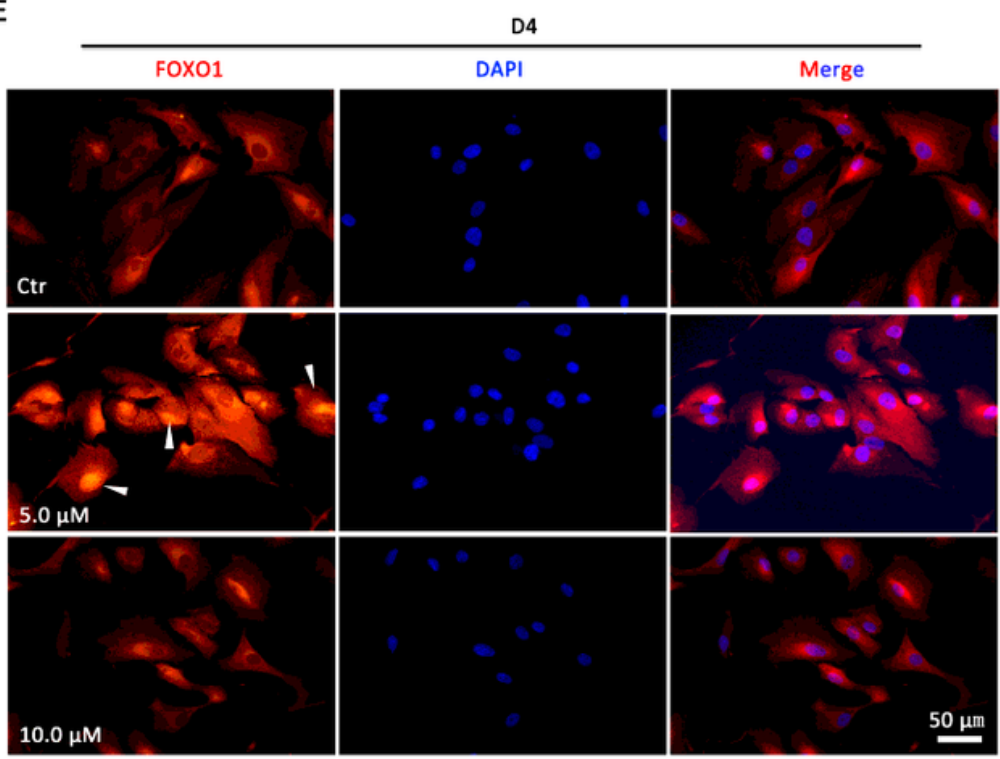

Figure 4 
The adrenergic signaling pathway down-regulate AKT activity, associated withincreased FOXO1 expression and nuclear localization. (A) Western blot analysis of total protein and active forms of PKA, ERK and AKT signaling pathways in D4 decidualized cell with different treatment; Epi, epinephrine; (B) Western blot analysis of ADRA2C and decidual development-related transcription factors HOXA10, STAT3 and FOX01. Epi, epinephrine; (C) Western blot analysis of FOX01 in the nucleus and cytoplasm fraction; $\mathrm{N}$, nucleus; C, cytoplasm; Epi, epinephrine; (D) Relative fold change in density levels for C. (E) Expression and localization of FOXO1 by cellular immunofluorescence assay. The arrow points out FOXO1 in the nucleus; Scale bars, $50 \mu \mathrm{M}$.

A

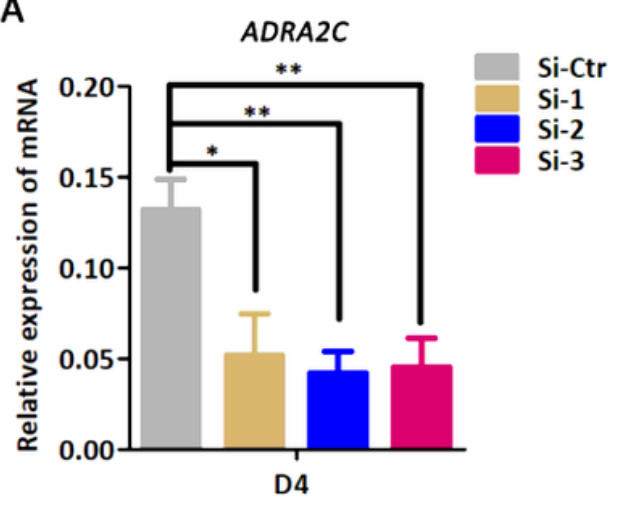

D

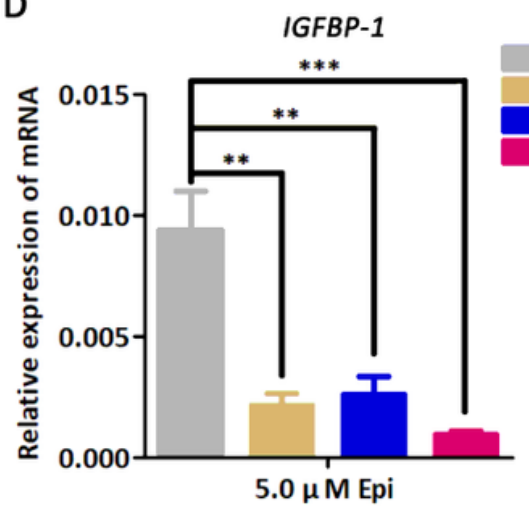

$\mathrm{F}$
B
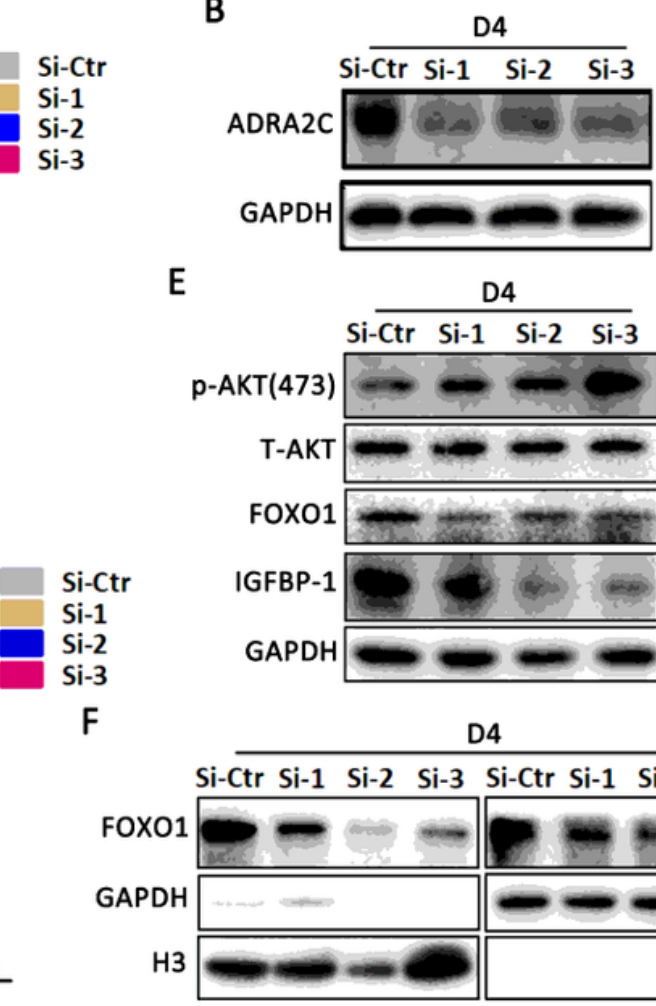

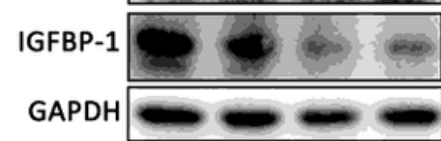

D4

Si-Ctr Si-1 Si-2 Si-3 Si-Ctr Si-1 $\quad$ Si-2 $\quad$ Si-3

\section{1}

$-$

H3

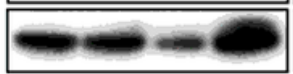

N
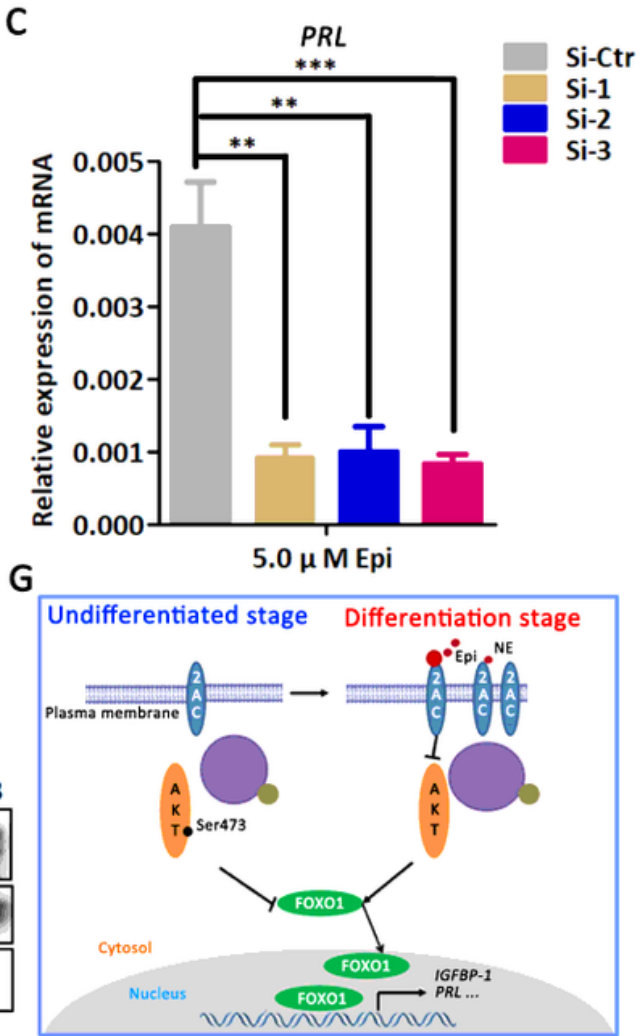

Figure 5

Knockdown of ADRA2C abolished the promoting effect of adrenergic signal-mediated decidualization of HESCs.(A, B) The mRNA and protein expression level for knockdown efficiency of ADRA2C in HESCs; $(C, D)$ The mRNA level for PRL and IGFBP-1 transcription in the presence of epinephrine after ADRA2C knockdown. Values are normalized to GAPDH expression level and indicate as the mean $\pm S E M, n=3$. Epi, epinephrine; (E) Western blot analysis of AKT and FOXO1 expression in the presence of epinephrine after knockdown of ADRA2C; (F) Western blot analysis of FOX01 expression in different cellular compartment after knockdown of ADRA2C. N, nucleus; C, cytoplasm; Epi, epinephrine; (G) Schematic diagram of ADRA2C mediated epinephrine signaling in HESCs during decidualization. 
A

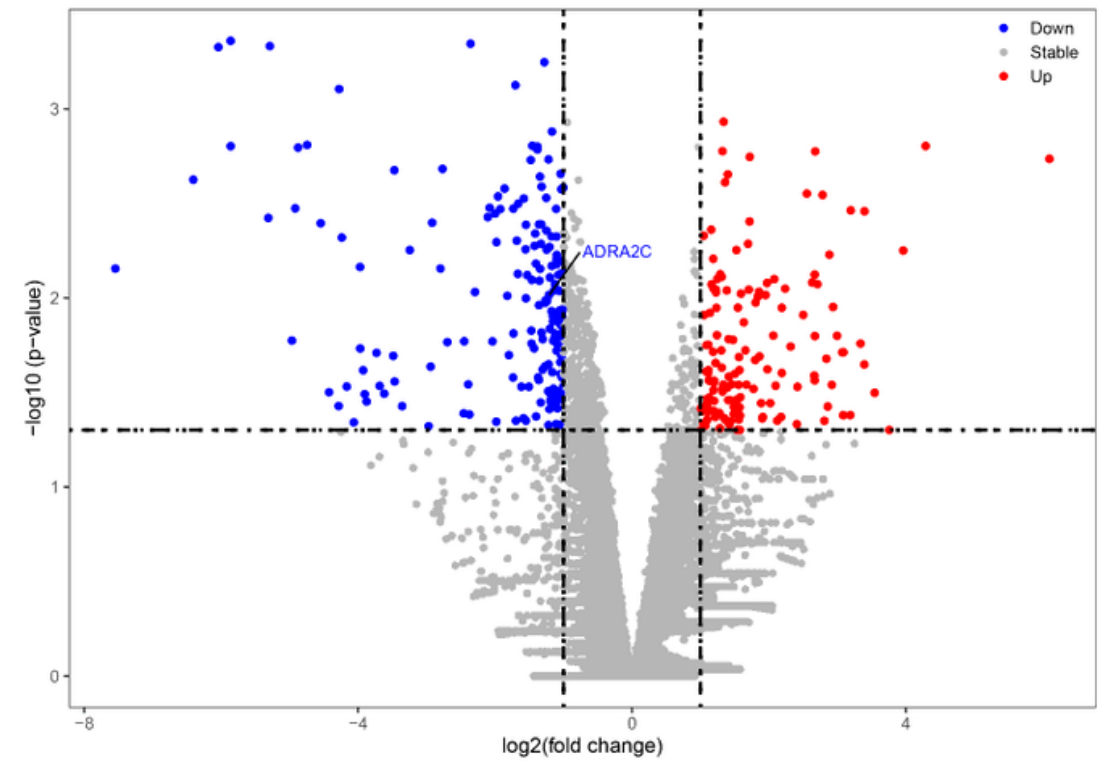

B

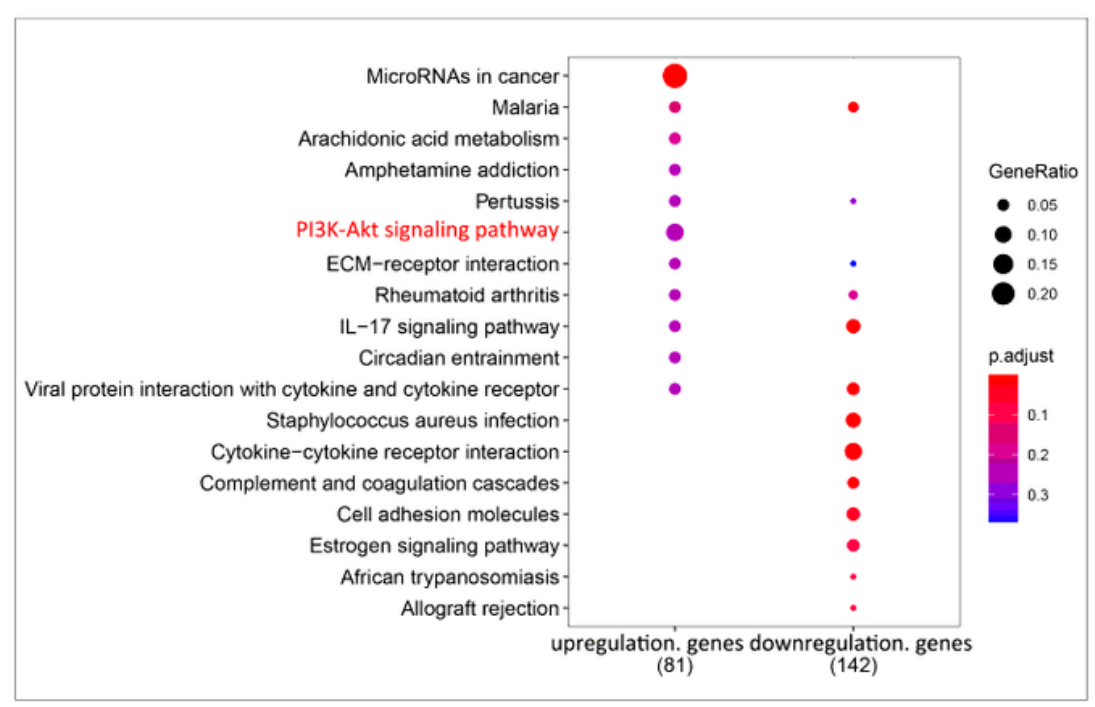

C

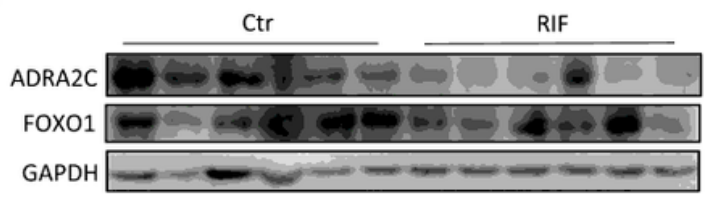

D

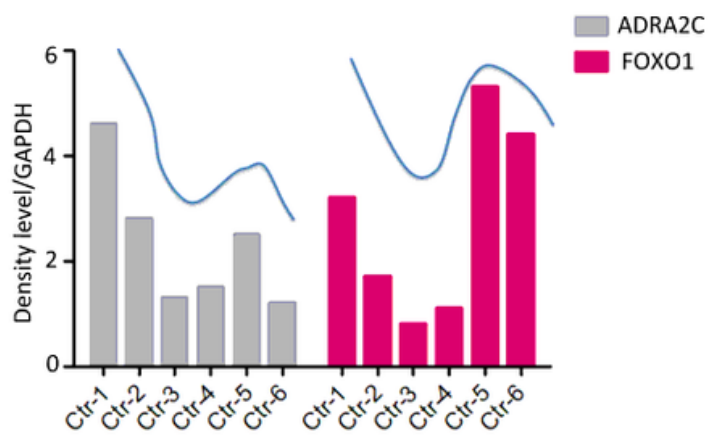

E

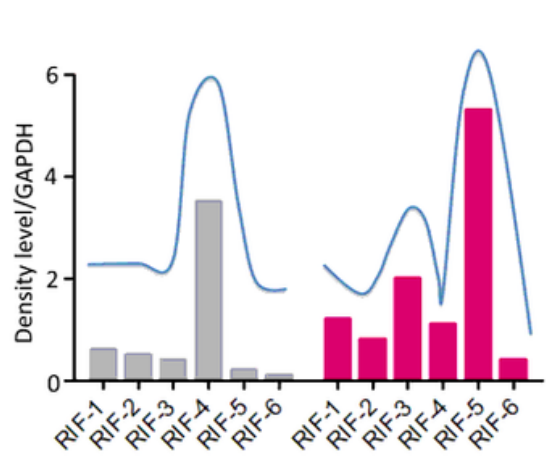

\section{Figure 6}

Alterations of ADRA2C expression patterns and signaling pathways in Mid-secretory endometrium with RIF compared with control. (A) Volcano plot of the log2 (fold change) and log10 (p-value). There are significant differences in RNA expression between RIF and control endometrial receptive tissues (upregulated genes in red and down-regulated genes in blue). (B) KEGG pathway analysis of significantly enriched genes and hub gene counts. For each term, the number of enriched genes is indicated by the dot size; while the level of significance is represented by the color. Blue indicates low significance while red represents high significance (adjust $P<0.5$ ). (C) Western blot analysis of ADRA2C and F0X01 expression in Mid-secretory phage endometrial tissues with RIF compared with control. (D, E) The relative proteins levels of ADRA2C and FOX01 in control and RIF for C. 
A

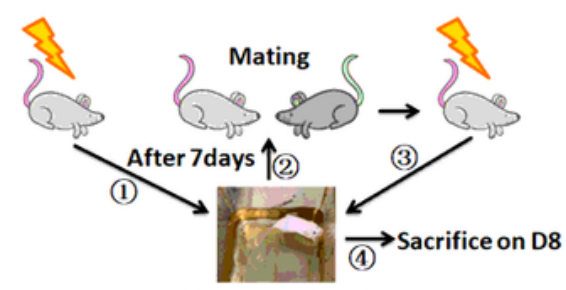

Cold water swimming

F
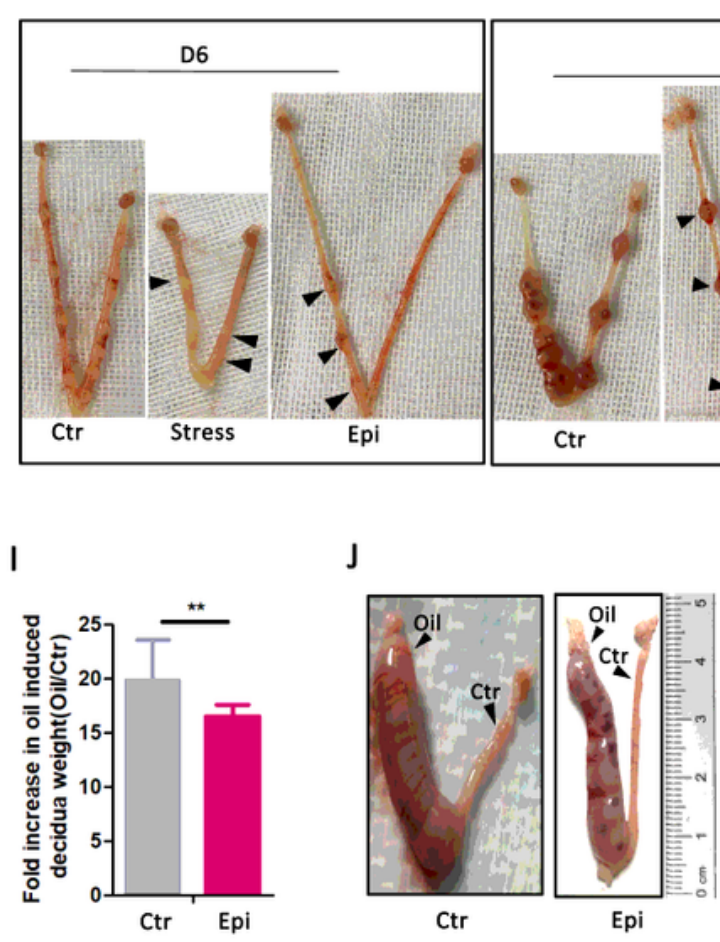

C

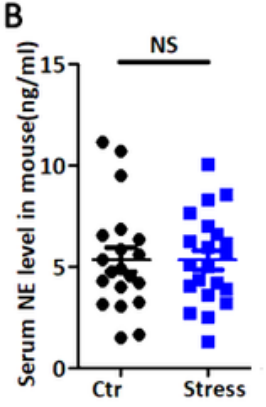

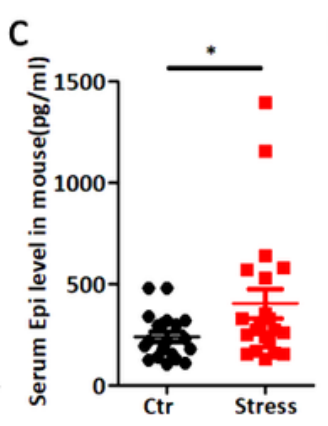

D

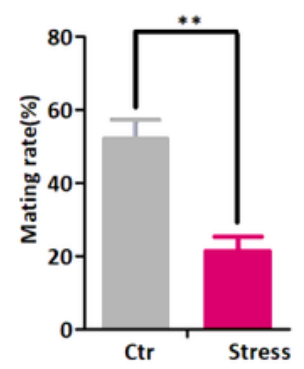

E

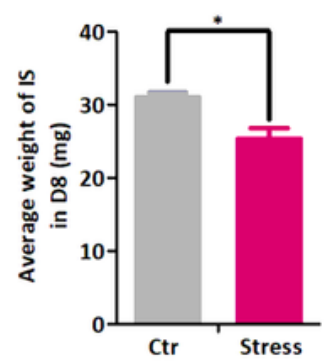

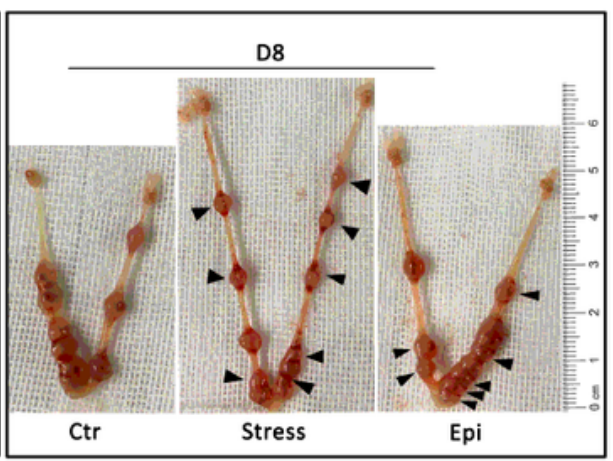

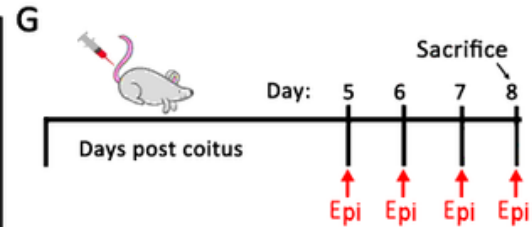

H
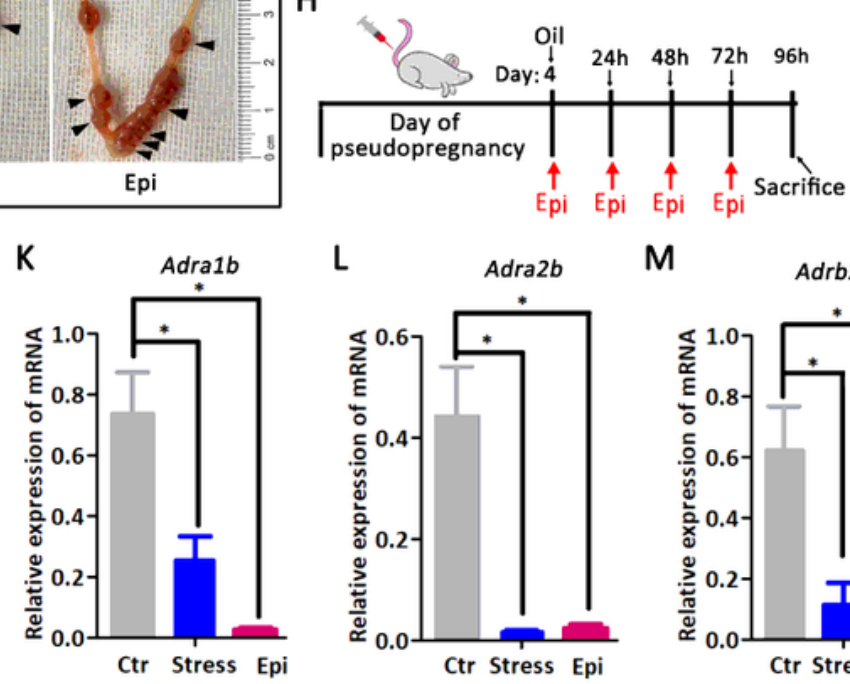

L
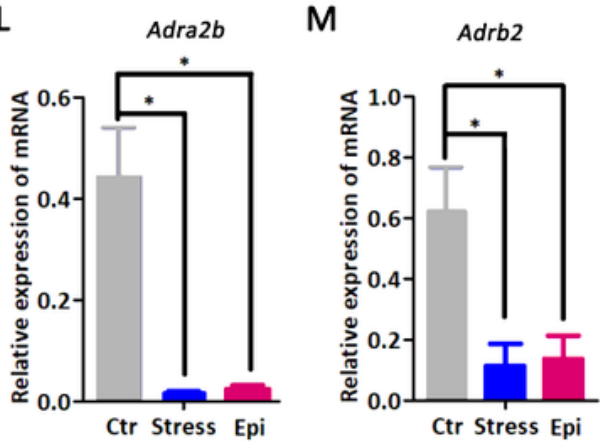

N

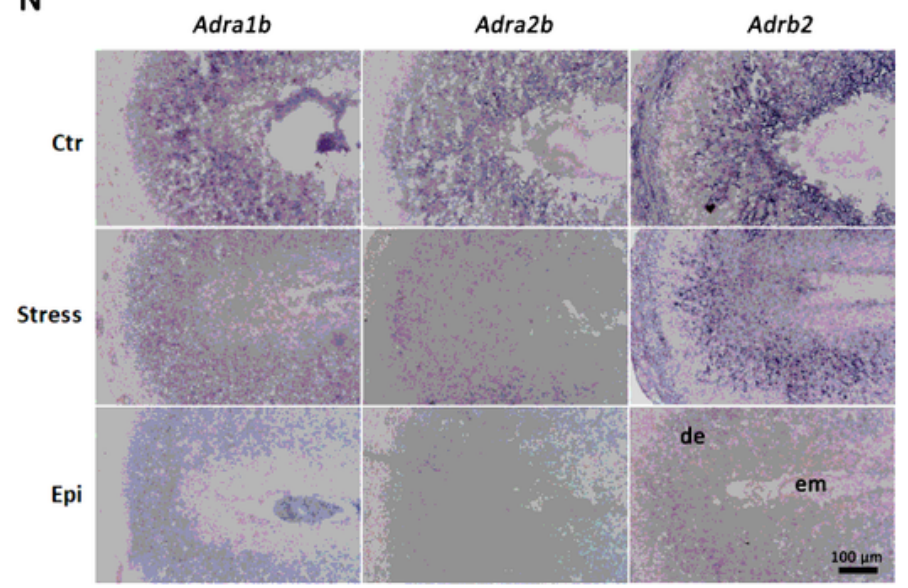

0

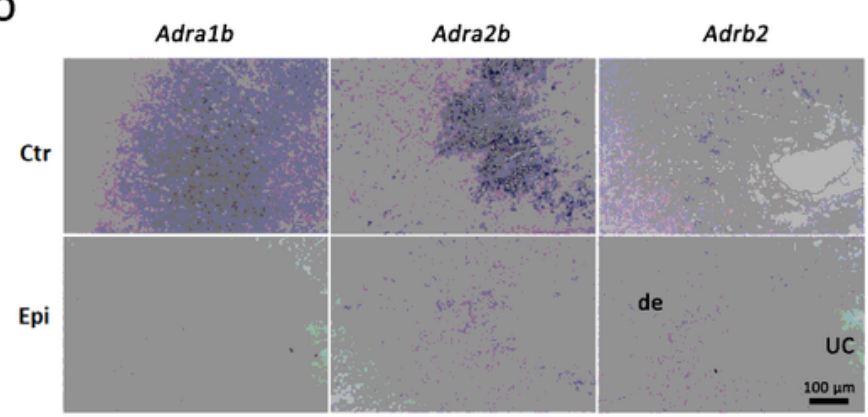

\section{Figure 7}

Exposure ofendogenous and exogenous epinephrine leads to changes in the endometrial adrenergic receptor expression in mice. (A) Schematic diagram of experimental design of stressed mouse model. $(B, C)$ Serum epinephrine and norepinephrine levels in stressedmice $(n=21)$ versus control mice $(n=20)$. (D) Mating rate of stress mice was significaltly decreased $(n=21)$ versus control mice $(n=20)$. (E) The average weight of IS was decreased in stress mice on D8. IS, implantation site. (F) Representative images of 
embryos in the uterus from stressed mice versus control on D6 and D8. The arrow points out growthrestricted implantation sites; (G) Schematic illustration of exogenous Epi exposure experimental design. (H) A schematic diagram of the experimental design for pseudopregnant mice exposed to exogenous Epi. (I)The fold increase after oil induced decidual weight. (J) Morphological differences of pseudopregnant uterus after $96 \mathrm{~h}$ of oil induction between vehicle and $100 \mu \mathrm{g} /$ mouse epinephrine treatment. Ctr, Nondecidualization; Oil, artificial decidualization; (K-M) qRT-PCR analyses of Adra1b, Adra2b and Adrb2 in the uteri on stress mouse and exogenous Epi exposure mouse model versus control. $(\mathrm{N})$ In situ hybridization for expression of Adra1b, Adra2b and Adrb2 in the uteri on stressed mouse and exogenous Epi exposure mouse model versus control. Scale bars, $100 \mu \mathrm{m}$; em, embryo; de, decidua; (0) In situ hybridization revealed the alternation expression of Adra1b, Adra2b and Adrb2 in the uteri on exogenous Epi exposure pseudopregnancy mouse model versus control. Scale bars, $100 \mu \mathrm{m}$; de, decidua; UC, uterine cavity.

\section{Supplementary Files}

This is a list of supplementary files associated with this preprint. Click to download.

- supplementaryfiles.docx 